\title{
COMPOSABLE TOPOLOGICAL PROPERTIES AND SEMIGROUPS OF RELATIONS
}

\author{
KENNETH D. MAGILL, Jr. \\ (Received 9 September 1968) \\ Communicated by G. B. Preston
}

\section{Introduction}

It is assumed throughout this paper that all topological spaces under consideration are Hausdorff. Since the notion of topological property is fundamental in this paper, we begin by making it precise. For our purposes here, it is sufficient to think of a topological property $Q$ as being a class of spaces such that if $X \in Q$ and $Y$ is homeomorphic to $X$, then $Y \in Q$. To say that a space $X$ has property $Q$ would then be equivalent to saying that $X \in Q$.

Definition (1.1). A topological property $Q$ is said to be composable if (1.1.1) all points have property $Q$ and

(1.1.2) if $X$ is any topological space and $\alpha, \beta \in X \times X$ have property $Q$, then $\alpha \circ \beta$ has property $Q$ where $\alpha \circ \beta$ is defined by

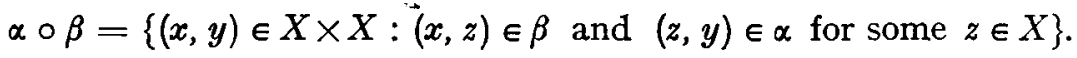

We see that the family of all binary relations on a topological space which have a composable property form a semigroup under composition as defined in (1.1.2) and it is these semigroups that we study in this paper. Composable topological properties are investigated in section 2 . It is shown that such a property must be pairwise productive. Consequently, for an example of a topological property which is not composable, one need only choose a property which is not pairwise productive such as normality. It is also observed in section 2 that connectedness is not a composable property. Since connectedness is productive, this implies that being composable and being pairwise productive are not equivalent. One of the most important productive properties is composable, however, and that is compactness. This is the content of Theorem (2.6).

In section 3, we investigate the semigroup, under composition, of all binary relations on a topological space $X$ (i.e., subsets of $X \times X$ ) which have a given composable topological property $Q$. The isomorphisms between 
one such semigroup and another are determined and, with sorne additional restrictions, the automorphism groups of such semigroups are determined. The special case where the composable property is compactness is treated in further detail.

\section{Composable topoiogical spaces}

Let $Q$ be any composable properiy and let $X$ be any space with at least two distince points $p$ and $q$. Then according to (1.1.1), both $\alpha=\{(p, p)\}$ and $\beta=\{(q, q)\}$ have properiy $Q$. It follows from (1.1.2) that $\phi=\alpha \circ \beta$ also has property $Q$. This proves

Proposirion (2.1). The empty set (or space) has property $Q$.

Proposíín (2.2). Any composable topological property is aiso pairwise productive.

Proof. Let $Q$ be any composable topological property and suppose that $X$ and $Y$ are two spaces which have property $Q$. Because of Proposition (2.1), we may assume that $X \times Y$ is nonempty. Choose any $(a, b) \in X \times Y$ and let

and

$$
\alpha=\{((a, b),(a, y)): y \in Y\}
$$

$$
\beta=\{((x, b),(a, b)): x \in X\} .
$$

Then $\alpha$ and $\beta$ are relations on $X \times Y$ (i.e., subsets of $(X \times Y) \times(X \times Y))$ which have property $Q$ since $\alpha$ is homeomor phic to $Y$ and $\beta$ is homeomorphic to $X$. It therefore follows from (1.1.2) that

$$
\alpha \circ \beta=\{((x, b),(a, y)): x \in X \text { and } y \in Y\}
$$

also has property $Q$. This concludes the proof since $\alpha \circ \beta$ is homeomorphic to $X \times Y$.

Since normality is not pairwise productive, the previous result immediately implies

Corollary (2.3). Normality is not a composable topological property.

The following result shows that one cannot drop the adjective pairwise in the statement of Proposition (2.2). The proof is straightforward and will not be given.

Proposition (2.4). Let $\lambda$ be any infinite cardinal number and let $Q_{\lambda}$ denote the property of 'consisting of less than $\lambda$ elements'. Then $Q_{\lambda}$ is a composable topological property which is not productive. 
Because of Proposition (2.2), we must look for composable properties among the pairwise productive properties. The following result shows, however, that we cannoc expect every pairwise productive (or even productive) property to be composable.

Proposition (2.5). Conneciedness is noi a composable property.

Proof. Let $R$ denote the real line and let

$$
\begin{aligned}
& \alpha=\{(0, x): x \in R\} \\
& \beta=\left\{(x, y) \in R \times R: x^{2}+y^{2}=1\right\} .
\end{aligned}
$$

Both $\alpha$ and $\beta$ are coinecred but

$$
\alpha \circ \beta=\{(-1, x): x \in R\} \cup\{(1, x): x \in R\}
$$

is not connected. Consequently, connectedness is not composable.

THeOREM (2.6). Compaciness is a composable topological property.

Proof. We prove this resuit using nets. Our terminology will be that of [2]. Let $X$ be any topoiogicai space and suppose that $\alpha$ and $\beta$ are compact subsets of $X \times X$. Let $\langle h, H\rangle$ denote any net in $\alpha \circ \beta$. That is, let $H$ be any directed set and let $h$ be any function which maps $H$ into $\alpha \circ \beta$. We will show that $\alpha \circ \beta$ is compact by constructing a subnet of $\langle h, H\rangle$ which converges to a point in $\alpha \circ \beta$. We recall that a net $\langle j, J\rangle$ is said to be a subnet of $\langle h, H\rangle$ if there exists a function $k$ mapping $J$ into $H$ such that

$$
j=h \circ k
$$

and

(2.6.2) for each $m \in H$, there exists an $n \in J$ such that if $p \geqq n$, then $k(p) \geqq m$.

Now, for each $n \in H, h(n)=\left(x_{n}, y_{n}\right) \in \alpha \circ \beta$ and there exists an element $z_{n} \in X$ such that $\left(x_{n}, z_{n}\right) \in \beta$ and $\left(z_{n}, y_{n}\right) \in \alpha$. Define a function $j$ mapping $H$ into $\beta$ by $j(n)=\left(x_{n}, z_{n}\right)$. Then $\langle j, H\rangle$ is a net in $\beta$ and since $\beta$ is compact, there exists a subnet $\langle l, L\rangle$ of $\langle j, H\rangle$ which converges to a point $(a, b) \in \beta$. Since $\langle l, L\rangle$ is a subnet of $\langle j, H\rangle$, there exists a function $k$ mapping $L$ into $H$ such that

$$
l=j \circ k
$$

and

(2.6.4) for each $m \in H$, there exists an $n \in L$ such that if $p \geqq n$, then $k(p) \geqq m$.

Now define a function $u$ from $L$ into $\alpha$ by $u(n)=\left(z_{k(n)}, y_{k(n)}\right)$. Then 
$\langle u, L\rangle$ is a net in $\alpha$ and since $\alpha$ is compact, there exists a subnet $\langle v, V\rangle$ which converges to a point $(c, d)$ in $\alpha$. Since $\langle v, V\rangle$ is a subnet of $\langle u, L\rangle$, there exists a function w mapping $V$ into $L$ such that

$$
v=u \circ w
$$

and

(2.6.6) for each $m \in L$, there exists an $n \in V$ such that if $p \geqq n$, then $w(p) \geqq m$.

Consider the net $\langle h \circ k \circ w, V\rangle$. Since the function $k$ satisfies (2.6.4) and $w$ satisfies (2.6.6), it follows that for each $n \in H$, there exists an $n \in V$ such that if $p \geqq n$, then $(k \circ w)(p) \geqq m$. Consequently, $\langle h \circ k \circ w, V\rangle$ is a subnet of $\langle h, H\rangle$. The proof will be complete when we show that $\langle h \circ k \circ w, V\rangle$ converges to $(a, d)$ and that $(a, d) \in \alpha \circ \beta$. First, let $\pi_{1}$ and $\pi_{2}$ denote the projection mappings from $X \times X$ into $X$ which are defined by $\pi_{1}(x, y)=x$ and $\pi_{2}(x, y)=y$ for all $(x, y) \in X \times X$. Using (2.6.3) and (2.6.5) one verifies that $\left\langle\pi_{1} \circ v, V\right\rangle$ is a subnet of $\left\langle\pi_{2} \circ l, L\right\rangle$. Since $\langle l, L\rangle$ converges to $(a, b),\left\langle\pi_{2} \circ l, L\right\rangle$ converges to $b$. Similarly, since $\langle v, V\rangle$ converges to $(c, d),\left\langle\pi_{1} \circ v, V\right\rangle$ converges to $c$. Since $\left\langle\pi_{1} \circ v, V\right\rangle$ is a subnet of $\left\langle\pi_{2} \circ l, L\right\rangle$, it follows that $b=c$ (recall that all spaces are assumed to be Hausdorff and hence a net can converge to at most one point). Consequently, $(b, d)=(c, d) \in \alpha$ and since $(a, b) \in \beta$, it follows that $(a, d) \in \alpha \circ \beta$. We must yet show that $\langle h \circ k \circ w, V\rangle$ converges to $(a, d)$. For this, it will be sufficient to show that the nets $\left\langle\pi_{1} \circ h \circ k \circ w, V\right\rangle$ and $\left\langle\pi_{2} \circ h \circ k w, V\right\rangle$ converge to $a$ and $d$ respectively. Using (2.6.3), one shows that

$$
\pi_{1} \circ h \circ k \circ w=\pi_{1} \circ l \circ w
$$

and it follows that $\left\langle\pi_{1} \circ h \circ k \circ w, V\right\rangle$ is a subnet of $\left\langle\pi_{1} \circ l, L\right\rangle$. Since $\langle l, L\rangle$ converges to $(a, b),\left\langle\pi_{1} \circ l, L\right\rangle$ must converge to $a$ and therefore $\left\langle\pi_{1} \circ h \circ k \circ w, V\right\rangle$ converges to $a$. On the other hand, it follows from (2.6.5) that $\pi_{2} \circ h \circ k \circ w=\pi_{2} \circ v$. Hence, the nets $\left\langle\pi_{2} \circ h \circ k \circ w, V\right\rangle$ and $\left\langle\pi_{2} \circ v, V\right\rangle$ are identical and must converge to $d$ since $\langle v, V\rangle$ converges to $(c, d)$. This completes the proof.

Proposition (2.7). Let $Q$ denote any composable topological property and let $\lambda$ denote any infinite cardinal number. Let $(Q, \lambda)$ denote the property of 'being the union of less than or equal to $\lambda$ subspaces, each having property $Q$ '. Then $(Q, \lambda)$ is a composable topological property.

Proof. Let $X$ be any topological space and let $\alpha$ and $\beta$ be two subsets of $X \times X$ with property $(Q, \lambda)$. Then there exist families of sets $\left\{K_{a}\right\}_{a \in A}$, $\left\{L_{b}\right\}_{b \in B}$ such that each $K_{a}$ and each $L_{b}$ has property $Q$, both card $(A)$ and card $(B)$ are less than or equal to $\lambda$ and 


$$
\begin{aligned}
& \alpha=\cup\left\{K_{a}\right\}_{a \in A} \\
& \beta=\cup\left\{L_{b}\right\}_{b \in B} .
\end{aligned}
$$

One verifies in a routine manner that

$$
\alpha \circ \beta=\cup\left\{K_{a} \circ L_{b}\right\}_{(a, b) \in A \times B} .
$$

Since each $K_{a} \circ L_{b}$ has property $Q$ and card $(A \times B) \leqq \lambda$, it follows that $\alpha \circ \beta$ has property $(Q, \lambda)$. Thus, $(Q, \lambda)$ is a composable topological property.

Let us recall that a space is defined to be $\sigma$-compact if it is the union of a countable number of compact subspaces. An application of Theorem (2.6) and Proposition (2.7) immediately yields

CoRollary (2.8). o-compactness is a composable topological property.

We remark that one can verify that sequential compactness is also a composable topological property.

\section{Semigroups of binary relations with composable properties}

Let $Q$ denote any composable topological property and let $X$ denote any topological space. According to (1.1.2), the family of all binary relations on $X$ which have property $Q$ is a semigroup under composition. We will denote this semigroup by $\mathscr{S}_{Q}[X]$. Our first result which concerns the semigroup $\mathscr{S}_{Q}[X]$ is essentially a translation of Theorem (2.2) of [4]. It will be convenient to recall that result along with some relevant definitions.

First of all, a triform was defined in [4] as a triple $\left(X, \mathscr{F}_{X}, \mathscr{S}[X]\right)$ where $X$ is a nonempty set, $\mathscr{F}_{X}$ is a family of subsets of $X, \mathscr{S}[X]$ is a family of subsets of $X \times X$ and the following conditions are satisfied:

(A) $\{(x, x)\} \in \mathscr{S}[X]$ for each $x \in X$.

(B) $H \in \mathscr{F}_{X}$ if and only if $H \times\{x\} \in \mathscr{S}[X]$ for some $x \in X$.

(C) $\alpha \circ \beta \in \mathscr{S}[X]$ for each $\alpha, \beta \in \mathscr{S}[X]$ where $\alpha \circ \beta$ is defined by $\alpha \circ \beta=\{(x, y) \in X \times X:(x, z) \in \beta$ and $(z, y) \in \alpha$ for some $z \in X\}$.

Because of condition (C), $\mathscr{S}[X]$ is a semigroup of binary relations on $X$ and is referred to in [4] as a triform semigroup. Theorem (2.2) is the main result of that paper. It describes all the isomorphisms from one triform semigroup onto another and is essentially as follows:

THEOREM (3.1). The following statements concerning a bijection $\varphi$ from a triform semigroup $\mathscr{S}[X]$ onto a triform semigroup $\mathscr{S}[Y]$ are equivalent:

(3.1.1) $\varphi$ is an isomorphism.

(3.1.2) There exists a bijection $h$ from $X$ onto $Y$ such that $h[A] \in \mathscr{F}_{Y}$ for each $A \in \mathscr{F}_{X}, h^{-1}[A] \in \mathscr{F}_{X}$ for each $A \in \mathscr{F}_{Y}$ and, furthermore, $\varphi(\alpha)=h \circ \alpha \circ h^{-1}$ for each $\alpha \in \mathscr{S}[X]$. 
(3.1.3) There exists a bijection $h$ from $X$ onto $Y$ such that $h[A] \in \mathscr{F}_{Y}$ for each $A \in \mathscr{F}_{X}, h^{-1}[A] \in \mathscr{F}_{X}$ for each $A \in \mathscr{F}_{Y}$ and. furthermore, $\varphi(\alpha)=\{(h(x), h(y)):(x, y) \in \alpha\}$ for each $\alpha \in \mathscr{S}[X]$.

Now let $X$ denote any topological space. We let $Q_{x}$ denote the family of all subsets of $X$ which have the composable property $Q$ and we recall that $\mathscr{S}_{Q}[X]$ denotes the semigroup, under composition. of all binary relations on $X$ which have property $Q$. Because of (1.1.1), $\{(x, x)\} \in \mathscr{S}_{Q}[X]$ for each $x \in X$. Furthermore, for any nonempty subset $H$ of $X$ and any $x \in X, H$ and $H \times\{x\}$ are homeomorohic. Thus, $H \in Q_{X}$ if and only if $H \times\{x\} \in \mathscr{S}_{Q}[X]$. Therefore, conditions (A), (B) and (C) are satisfied and the triple $\left(X, Q_{X}, \mathscr{S}_{Q}[X]\right)$ is a triform. Before stating our first result on semigroups of the type $\mathscr{S}_{\mathcal{Q}}[X]$, it will be convenient to have the following

DFFinition (3.2). Let $Q$ be any topological property. A bijection $h$ from a topological space $X$ onto a topological space $Y$ is a $Q$-morphism if $h[A]$ has property $Q$ whenever $A \subset X$ has property $Q$ and, similarly, $h^{-1}[A]$ has property $Q$ whenever $A \subset Y$ has property $Q$.

With all this in mind. we see that Theorem (3.1) translates into

THEORFM (3.3). I.et $Q$ be any composable topological property and let $\mathscr{S}_{Q}[X]$ and $\mathscr{S}_{Q}[Y]$ denote the semigroups of all binary relations on $X$ and $Y$ respectively which have property $Q$. Then the following statements concerning a bijection $\varphi$ from $\mathscr{S}_{Q}[X]$ onto $\mathscr{S}_{Q}[Y]$ are equivalent:

(3.3.1) $\varphi$ is an isomorphism.

(3.3.2) There exists a Q-morphism $h$ from $X$ onto $Y$ such that $\varphi(\alpha)=h \circ \alpha \circ h^{-1}$ for each $\alpha \in \mathscr{S}_{Q}[X]$.

(3.3.3) There exists a Q-morbhism $h$ from $X$ onto $Y$ such that $\varphi(\alpha)=\{(h(x), h(y)):(x, y) \in \alpha\}$ for each $\alpha \in \mathscr{S}_{Q}[X]$.

THEOREM (3.4). For any composable topological property $Q$ and any topological space $X$, the automorphism group of $\mathscr{S}_{Q}[X]$ is isomorphic to a subgroup of the group, under composition, of all Q-morphisms which map $X$ onto $X . I f$, in addition to being composable. $Q$ is also hereditary and preserved by continuous mappings, then the automorphism group of $\mathscr{S}_{Q}[X]$ is isomorphic to the group of all $Q$-morphisms rehich map $X$ onto $X$.

ProOF. Let $\mathscr{A}$ denote the automorphism group of $\mathscr{I}_{Q}[X]$ and let $\mathscr{G}$ denote the group of all $Q$-morphisms which map $X$ onto $X$. For each $\varphi \in \mathscr{A}$, there exists, according to Theorem (3.3), a $Q$-morphism $h$ mapping $X$ onto $X$ such that

$$
\varphi(\alpha)=h \circ \alpha \circ h^{-1}
$$

for each $\alpha \in \mathscr{S}_{Q}[X]$. This $Q$-morphism is uniaue and we define a mapping $\Phi$ 
from $\mathscr{A}$ into $\mathscr{G}$ by $\Phi(\varphi)=h$. Suppose $\Phi\left(\varphi_{1}\right)=h_{1}$ and $\Phi\left(\varphi_{2}\right)=h_{2}$. Then for any $\alpha \in \mathscr{S}_{Q}[X]$, we have

$$
\begin{aligned}
\left(\varphi_{1} \circ \varphi_{2}\right)(\alpha) & =\varphi_{1}\left(h_{2} \circ \alpha \circ h_{2}^{-1}\right) \\
& =h_{1} \circ h_{2} \circ \alpha \circ h_{2}^{-1} \circ h_{1}^{-1}=\left(h_{1} \circ h_{2}\right) \circ \alpha \circ\left(h_{1} \circ h_{2}\right)^{-1} .
\end{aligned}
$$

Thus $\Phi\left(\varphi_{1} \circ \varphi_{2}\right)=h_{1} \circ h_{2}=\Phi\left(\varphi_{1}\right) \circ \Phi\left(\varphi_{2}\right)$ which verifies the fact that $\Phi$ is a homomorphism. To see that $\Phi$ is injective, suppose that $\Phi(\varphi)=i$, the identity $Q$-morphism. Then $\varphi(\alpha)=i \circ \alpha \circ i^{-1}=\alpha$ for each $\alpha \in \mathscr{S}_{Q}[X]$ and this implies that the kernel of $\Phi$ consists of the identity.

Now assume that, in addition to being composable, $Q$ is hereditary and continuous images of spaces with property $Q$ also have property $Q$. Let $h$ be any element of $G$. We assert that if $\alpha \in \mathscr{S}_{Q}[X]$, (i.e., $\alpha$ has property $Q$ ), then, $h \circ \alpha \circ h^{-1} \in \mathscr{S}_{Q}[X]$. Since continuous images of $\alpha$ have property $Q$ and the domain, $\mathscr{D}(\alpha)$ and the range, $\mathscr{R}(\alpha)$ are continuous images of $\alpha$ under the projection mappings, it follows that both $\mathscr{D}(\alpha)$ and $\mathscr{R}(\alpha)$ have property $Q$. Since $\alpha \subset \mathscr{D}(\alpha) \times \mathscr{R}(\alpha)$, it follows that

$$
h \circ \alpha \circ h^{-1} C h \circ[\mathscr{D}(\alpha) \times \mathscr{R}(\alpha)] \circ h^{-1}=h[\mathscr{D}(\alpha)] \times h[\mathscr{R}(\alpha)] .
$$

Since $h$ is a $Q$-morphism, both $h[\mathscr{D}(\alpha)]$ and $h[\mathscr{R}(\alpha)]$ have property $Q$ and it follows from Proposition (2.2) that $h[\mathscr{D}(\alpha)] \times h[\mathscr{R}(\alpha)]$ has property $Q$. This implies that $h \circ \alpha \circ h^{-1}$ has property $Q$ since $Q$ is hereditary. Hence, $h \circ \alpha \circ h^{-1} \in \mathscr{S}_{Q}[X]$. It follows that the mapping defined by

$$
\varphi(\alpha)=h \circ \alpha \circ h^{-1}
$$

is an automorphism of $\mathscr{S}_{\mathrm{Q}}[X]$ and $\Phi(\varphi)=h$. Thus. in this particular case, $\Phi$ is an isomorphism from $\mathscr{A}$ onto $\mathscr{G}$.

The additional assumptions that $Q$ is hereditary and preserved under continuous mappings were used to prove that if $h$ is any $Q$-morphism from $X$ onto $X$, then $h \circ \alpha \circ h^{-1}$ has property $Q$ whenever $\alpha \subset X \times X$ has property $Q$. We do not know if these additional assumotions are needed to prove this. although we suspect that they are. We do give an example of a $Q$-morphism $h$ from $X$ onto $X$ such that $h \circ \alpha \circ h^{-1}$ does not have property $Q$ even though $\alpha \subset X \times X$ does. This particular property $Q$, however, is not composable.

Example (3.5). I.et $Q$ be the property of 'having more than one limit point'. Let $X$ denote the usual two-point compactification of the integers with $a$ being the limit point of the negative integers and $z$ being the limit point of the positive integers. Define a mapping $h$ from $X$ onto $X$ by

$$
h(a)=z, h(z)=a \text { and } h(n)=n \text { for } n \neq a, z .
$$

One easily verifies that $h$ is a $Q$-morphism. Let

$$
\alpha=\{(1,1),(1,2), \cdots(1, z)\} \cup\{(2,1),(2,2), \cdots(2, z)\} .
$$


Then $\alpha$ has property $Q$ since it has two limit points, namely $(1, z)$ and $(2, z)$. However,

$$
\begin{aligned}
h \circ \alpha \circ h^{-1} & =\{(h(x), h(y)):(x, y) \in \alpha\} \\
& =\{(1,1),(1,2), \cdots\} \cup\{(2,1),(2,2), \cdots\} \cup\{(1, a),(2, a)\}
\end{aligned}
$$

has no limit points.

As we mentioned previously, $Q$ is not composable (although it is productive). In fact, $Q$ does not even satisfy condition (1.1.1). This can be remedied by defining $Q$ in such a manner that all finite subsets have property $Q$. A more serious failing is that condition (1.1.2) is not satisfied. To see this, let

$$
\begin{aligned}
\beta_{1} & =\{(1,1),(2,2),(3,3), \cdots\}, \\
\beta_{2} & =\{(-1,1),(-1,2),(-1,3), \cdots(-1, z)\}, \\
\beta_{3} & =\{(-2,1),(-2,2),(-2,3), \cdots(-2, z)\}, \\
\gamma & =\beta_{1} \cup \beta_{2} \cup \beta_{3}
\end{aligned}
$$

and note that

$$
\gamma \circ \alpha=\{(1,1),(1,2)(1,3), \cdots\} \cup\{(2,1),(2,2),(2,3), \cdots\} .
$$

It is not difficult to show that if $h$ is a homeomorphism from $X$ onto $Y$ and $Q$ is any topological property, then $h \circ \alpha \circ h^{-1} \subset Y \times Y$ has property $Q$ if $\alpha \subset X \times X$ has property $Q$. Define a mapping $k$ from $X \times X$ onto $Y \times Y$ by

$$
k(x, y)=(h(x), h(y)) .
$$

The desired conclusion is a consequence of the fact that $k$ is a homeomorphism and

$$
h \circ \alpha \circ h^{-1}=\{(h(x), h(y)):(x, y) \in \alpha\}=k[\alpha] .
$$

Since we have need to refer to this observation later, it is convenient to state it formally as

Proposition (3.6). Let $Q$ be any topological property and let $h$ be any homeomorphism from a space $X$ onto a space $Y$. Then $h \circ \alpha \circ h^{-1} \subset Y \times Y$ has property $Q$ whenever $\propto \subset X \times X$ has property $Q$.

We now consider the special case where the composable topological property is compactness. We denote this property by $K$ and we denote the semigroup, under composition, of all compact binary relations on a topological space $X$ by $\mathscr{S}_{K}[X]$. It will be convenient, during the discussion of semigroups of compact relations, to restrict ourselves to $k$-spaces [2, p. $230-231]$. These are defined to be those spaces with the property that any subset which intersects each compact subset in a closed set must, itself, be closed. The class of $k$-spaces includes all locally compact spaces and all first countable spaces $[2$, p. 231, Theorem 13]. In particular, every metric 
space is a $k$-space. One readily shows that if $X$ and $Y$ are $k$-spaces, then any $K$-morphism from $X$ onto $Y$ is, in fact, a homeomorphism. This, together with Theorem (3.3) results in

THEOREM (3.7). Let $X$ and $Y$ be $k$-spaces and let $\mathscr{S}_{K}[X]$ and $\mathscr{S}_{K}[Y]$ denote the semigroups of compact binary relations on $X$ and $Y$ respectively. Then the following statements concerning a bijection $\varphi$ from $\mathscr{I}_{K}[X]$ onto $\mathscr{S}_{\boldsymbol{K}}[Y]$ are equivalent:

(3.7.1) $\varphi$ is an isomorphism.

(3.7.2) There exists a homeomorphism $h$ from $X$ onto $Y$ such that $\varphi(\alpha)=h \circ \alpha \circ h^{-1}$ for each $\alpha \in \mathscr{I}_{\boldsymbol{K}}[X]$.

(3.7.3) There exists a homeomorphism $h$ from $X$ onto $Y$ such that $\varphi(\alpha)=\{(h(x), h(y)):(x, y) \in \alpha\}$ for each $\alpha \in \mathscr{S}_{K}[X]$.

CoROLlary (3.8). Two k-spaces $X$ and $Y$ are homeomorphic if and only if $\mathscr{S}_{K}[X]$ and $\mathscr{S}_{K}[Y]$ are isomorphic.

PRoof. If $\mathscr{S}_{K}[X]$ and $\mathscr{S}_{K}[Y]$ are isomorphic, it follows immediately from Theorem (3.7) that $X$ and $Y$ are homeomorphic. On the other hand, if $h$ is a homeomorphism from $X$ onto $Y$, we can define a mapping $\varphi$ by $\varphi(\alpha)=h \circ \alpha \circ h^{-1}$. It follows from Proposition (3.6) that $\varphi$ maps $\mathscr{S}_{K}[X]$ bijectively onto $\mathscr{S}_{K}[Y]$ and hence that $\varphi$ is an isomorphism.

Corollary (3.9). Let $X$ be a $k$-space. Then the automorphism group of $\mathscr{S}_{K}[X]$ is isomorphic to the group, under composition, of all homeomorphisms which map $X$ onto $X$.

PROOF. Let $\mathscr{A}$ denote the automorphism group of $\mathscr{S}_{K}[X]$ and let $\mathscr{G}$ denote the group of homeomorphisms on $X$. By Theorem (3.7) there exists, for any $\varphi \in \mathscr{A}$, a homeomorphism $h$ mapping $X$ onto $X$ which satisfies (3.7.2). This homeomorphism is unique and we define a mapping $\Phi$ from $\mathscr{A}$ into $\mathscr{G}$ by $\Phi(\varphi)=h$. Just as in the proof of Theorem (3.4), one shows that $\Phi$ is a monomorphism. It follows from Proposition (3.6) that if $t$ is any homeomorphism mapping $X$ onto $X$, then the mapping $\varphi_{t}$, defined by $\varphi_{t}(\alpha)=t \circ \alpha \circ t^{-1}$, belongs to $\mathscr{A}$. Since $\Phi\left(\varphi_{t}\right)=t$, we conclude that $\Phi$ is surjective.

We remark that we were prevented from using Theorem (3.4) to prove the latter corollary by the fact that compactness is not hereditary.

An endomorphism $\varphi$ of a semigroup $S$ is defined to be inner if there exist elements $a$ and $b$ in $S$ such that $q(x)=a x b$ for each $x \in X$. M. L. Vitanza has shown [5, p. 1079, Theorem 1] that if such an endomorphism is surjective, then $S$ has an identity and $a$ and $b$ are inverses of each other relative to that identity. This implies, among other things, that any inner 
epimorphism is, in fact, an automorphism. Concerning the automorphisms of $\mathscr{S}_{K}[X]$, we have

THEOREM (3.10). The following statements about a $k$-space $X$ are equivalent:

(3.10.1) $X$ is compact.

(3.10.2) Every automorphism of $\mathscr{S}_{\boldsymbol{K}}[X]$ is inner.

(3.10.3) At least one automorphism of $\mathscr{S}_{K}[X]$ is inner.

(3.10.4) $\mathscr{S}_{K}[X]$ has an identity.

Proof. Suppose $X$ is compact and let $\varphi$ be an automorphism of $\mathscr{S}_{K}[X]$. Then by Theorem (3.7), there exists a homeomorphism $h$ from $X$ onto $X$ such that

$$
\varphi(\alpha)=h \circ \alpha \circ h^{-1}
$$

for each $\alpha \in \mathscr{S}_{K}[X]$. We regard $h$ as a subset of $X \times X$ and note that the mapping $H$ defined by

$$
H(x)=(x, h(x))
$$

is a homeomorphism from $X$ onto. $h$. Thus, $h$ belongs to $\mathscr{S}_{k}[X]$. In a similar manner, $h^{-1}$ does also and we conclude that $\varphi$ is an inner automorphism. Thus (3.10.1) implies (3.10.2). It is evident that (3.10.2) implies (3.10.3) and in view of the result of Vitanza that we mentioned previously, (3.10.3) implies (3.10.4). Therefore, we need only show that (3.10.4) implies (3.10.1). Suppose $\mathscr{S}_{K}[X]$ has an identity $I$. Then for any $(x, y) \in I,\{(x, y)\}$ belongs to $\mathscr{S}_{K}[X]$ and since $(x, y) \in\{(y, y)\} \circ I=\{(y, y)\}$, it follows that $x=y$. On the other hand, for any $x \in X,\{(x, x)\}=\{(x, x)\} \circ I$ which implies that $(x, x) \in I$. Therefore,

$$
I=\{(x, x): x \in X\} \text {. }
$$

Since $I \in \mathscr{S}_{K}[X]$, it is compact and since $X$ is the image of $I$ under either projection mapping, it too must be compact.

According to this latter result, if $X$ is not compact, then $\mathscr{S}_{K}[X]$ has no identity. However, each finite subset $\mathscr{T}$ of $\mathscr{S}_{K}[X]$ has an infinite number of identities. To see this, let $H$ be the union of the domains and the ranges of all of the relations in $\mathscr{T}$. Since both the domain and the range of a compact relation are compact, $H$ is the finite union of compact sets and hence, must also be compact. Since $X$ is not compact, $X-H$ must be infinite. For each $p \in X-H$, define

$$
I_{p}=\{(x, x) \in X \times X: x \in H\} \cup\{(p, p)\} .
$$

One readily verifies that each $I_{p}$ is an identity for each element of the subset $\mathscr{T}$. 
We conclude by mentioning that the substance of Corollary (3.8) is that within the class of $k$-spaces, the topological structure of a space $X$ determines and is determined by the algebraic structure of its semigroup $\mathscr{S}_{K}[X]$ of compact binary relations. Consequently, it would seem to be fruitful to investigate further the relationships between the two structures. We already know, for example, that $X$ is compact if and only if every automorphism of $\mathscr{S}_{K}[X]$ is inner. One would expect to find other such results relating topological properties of $X$ to algebraic properties of $\mathscr{S}_{\boldsymbol{K}}[X]$.

\section{References}

[1] A. H. Clifford and G. B. Preston, The algebraic theory of semigroups. Mathematical Surveys, Number 7 (Amer. Math. Soc., 1961)

[2] J. L. Kelley, General Topology, Van Nostrand, Princeton, 1955.

[3] E. S. Ljapin, Semigroups, Vol. 3, Translations of Mathematical Monographs (Amer. Math. Soc., 1963).

[4] K. D. Magill, Jr., 'Isomorphisms of triform semigroups', J. Austr. Math. Soc. 10 (1969), $185-193$.

[5] M. L. Vitanza, 'Mappings of semigroups associated with ordered pairs', Amer. Math. Monthly 73, (1966), 1078-1082.

State University of New York at Buffalo and University of Leeds 